\title{
Paradoxe Mutationen? Zu Gattungsinterferenzen im Minnesang: Tagelied-Wechsel und Pastourelle-Tagelied
}

\author{
Paradox Mutations: Genre-Interferences in Minnesang, shown \\ in the Example of Tagelied-Wechsel and Pastourelle-Tagelied
}

Kristýna Solomon

\begin{abstract}
The majority of the medieval poetry-(sub)genres has already been established in the classical period. The 'mainstream' genres coexisted with the so called hybrid texts, which often united two different subgenres within one song. In this paper I focus on examples of such mutations/ interferences, which combine two specific genres that can be positioned into a different spot within the time/ space-specter, such as an early combination of the domestic Wechsel with a dawn song by Morungen or a later down song/ pastourelle by an anonymous Czech author. In accordance with Eco's theory on open texts and Bachtin's idea of polyphony, the extent to which multivocalism represents an intended strategy of the producer of a lyrical text is to be discussed.
\end{abstract}

\section{Keywords}

dawn song; Wechsel; Minnesang; hybrid texts; multivocalism 
In dem monumentalen Buch Schwierige Modernität ${ }^{1}$ hat sich Kiening unter anderem mit der Paradoxie der Grenzerscheinungen beschäftigt, insbesondere im Hinblick auf die Darstellung des Todes im Mittelalter, welche am Anschaulichsten durch die Analyse des im Ackermann agierenden Todes präsentiert worden ist. Die Schwellenexistenz des Todes, der zwischen Sein und Nicht-Sein zu verorten ist, trotz dessen Nicht-Präsenz im Dialog präsent ist, der als eine Vermittlungsinstanz zwischen Diesseits und Jenseits zu verstehen ist, hat mich zur folgenden Überlegung angeregt:

Wie betrachtete der mittelalterliche Leser/ Hörer Elemente, oder auch Textstrukturen, welche in solchen Zwischenräumen schweben? Entsprach der damalige Erfahrungshorizont den binären Oppositionen schwarz/ weiß, hier/ da, draußen/ drinnen?

Es muss an dieser Stelle nicht akzentuiert werden, dass Gattungen Konstrukte der modernen Philologie sind, die sich auf das mehr oder minder authentische Gattungsverständnis beruft. ${ }^{2}$ Man darf jedoch annehmen, dass eine bestimmte Gattung über Parameter disponiert, welche diese von Repräsentanten anderer Gattungen unterscheidet und welche vom zeitgenössischen Leser/ Hörer enkodiert werden konnten³ (Systemreferenz, Architextualität). Jedoch stellen die mittelalterlichen lyrischen Gattungen äußerst dynamische Komplexe dar. So mutierte beispielsweise der im Frühen Minnesang etablierte einheimische Wechsel im Laufe der Zeit markant. Das einst als monologisch zu definierende Gebilde wanderte nicht nur auf der Skala zwischen monologisch - dialogisch $^{4}$, sondern auch hinsichtlich der Abgrenzung von den - auf den ersten Blick gravierend unterschiedlichen - Liedtypen. ${ }^{5}$

1 Kiening, Christian (1998): Schwierige Modernität. Der „Ackermann“ des Johannes von Tepl und die Ambiguität historischen Wandels. Tübingen: Niemeyer.

2 Jauß, Hans Robert (1972): Theorie der Gattungen und Literatur des Mittelalters. In: Jauß, Hans Robert Köhler Erich (hrsg.): Grundriss der romanischen Literaturen des Mittelalters, Bd. 1. Heidelberg, S. 107-138.

3 Bei den von mir zu besprechenden Texten gehe ich davon aus, dass die etablierten Gattungen dem mittelalterlichen Publikum dermaßen bekannt waren, dass es imstande war, ggf. die Divergenzen von der Norm wahrzunehmen. Das Gattungsparadigma verstehe ich als die notwendige „Verständnisfolie“, vgl. Eikelmann, Manfred (1999): Dialogische Poetik. Zur Kontinuität älterer poetologischer Traditionen des Minnesangs am Beispiel des Wechsels. In: Cramer, Thomas - Kasten, Ingrid (hrsg.): Mittelalterliche Lyrik. Probleme der Poetik, S. 85-106 (hier S. 104).

4 Der hohe Grad der Responsivität und formalen Verkettung der einzelnen Strophen signalisieren die Nähe zum Dialog; die Interreferenz einzelner Texte, die den Minnesang als Kettenkunst definieren, sind auch ein Beleg für den dialogischen Charakter (vgl. Reimar-Walther Fehde MF 112,23).

5 Dazu s. Schnell, Rüdiger (1999): Frauenlied, Manneslied und Wechsel im deutschen Minnesang. Überlegungen zu „gender“ und Gattung. In: Zeitschrift für deutsches Altertum und deutsche Literatur 128, S. 127180. Schnell wies auf den Mutationscharakter des Wechsels und dessen Annäherungstrend an das Manneslied hin, S. 142. Zum dialogischen Charakter des Wechsels vgl. Kerth, Sonja (2007): „Jô enwas ich niht ein eber wilde“. Geschlechterkonzeptionen im „Wechsel“. In: Zeitschrift für deutsches Altertum und deutsche Literatur 123, S. 143-161. Es wird darauf hingewiesen, dass die „Scheindialogizität“ des Wechsels von einer „echten“ Dialogizität kaum abzugrenzen ist, S. 147. 
In den folgenden Zeilen werde ich auf Lieder fokussieren, welche zwei anscheinend unvereinbare Gattungen verbinden, und versuche Ecos Konzept der open texts ${ }^{6}$ und Bachtins Multivokalität ${ }^{7}$ auf der produktiven Ebene zu überprüfen. ${ }^{8}$

\section{Tagelied-Wechsel Heinrichs von Morungen $(143,22)$}

Dem ausschließlich in der Handschrift $\mathrm{C}$ tradierten vierstrophigen Lied wird in der Forschung viel Aufmerksamkeit gewidmet. Die einmalige Harmonie von Form und Inhalt, sowie die kühne Kombination von Gattungen markieren den Ton als genuine Schöpfung Morungens. ${ }^{9}$

Der zu besprechende Tagelied-Wechsel Heinrichs verbindet zwei Gattungen, welche hinsichtlich der Raum-/ Zeit-Perspektive diametral verschieden sind. Im Folgenden wird der Text im Hinblick auf diese Problematik untersucht.

Das Lied wird mit dem Vers „Owê, / sol aber mir iemer mê“ eröffnet, der sich später als Refrain jeder Strophe entpuppt. Die in der ersten Strophe konzentrierten Bilder bedienen sich der gängigen Tagelied-Semantik. Dabei spielt das optische Wahrnehmen $\left(\right.$ Poetik des schouwens ${ }^{10}$ ) traditionsgemäß eine dominante Rolle, indem die „Lichterzeuger“ erwähnt werden: der in der Nacht leuchtende Körper, der „wîser danne ein snê“ (V. 4) sei, und der Mond. ${ }^{11}$ Das Subjekt der Rede, das männliche Ich, wird erst infolge des betrachteten Objekts erkennbar. Es ist nicht uninteressant, dass die Kausalität zugunsten der Relevanz umgekehrt wird. Der Sänger präsentiert an erster Stelle den Körper der Dame, welcher nicht sekundär, d.h. als Projektionsfläche des Mondscheins das Zimmer beleuchtet, sondern der mit dem Mondschein identifiziert wird. Trotz der signalisierten Subjektivität („ich wânde“) kann hier dem Objekt dessen außerordentliche Relevanz nicht abgesprochen werden:

ich wânde, ez solde sîn

des liehten mânen schîn. (Str. 1, V. 7-8)

6 Eco, Umberto (1979): The Role of the Reader. Explorations in the Semiotics of Texts. Bloomington: Indiana University Press.

7 Bachtin Michail (1979): Die Ästhetik des Wortes. Hrsg. von Rainer Grübel. Frankfurt am Main: Suhrkamp.

8 Eco definiert die Offenheit von Texten rezeptionsästhetisch. Ich setze mich mit Texten auseinander, welche mit der Zweischichtigkeit bereits in der produktiven Phase operieren.

9 Backes, Martina (hrsg.) (1992): Tagelieder des deutschen Mittelalters. Stuttgart: Reclam, S. 240.

10 Kasten, Ingrid (1986): Frauendienst bei Trobadours und Minnesängern im 12. Jahrhundert. Heidelberg: Carl Winter Universitätsverlag, S. 319.

11 Zu den Anklängen an Mariensymbolik vgl. Gottzmann, Carola L. (1989): Das Tagelied Heinrichs von Morungen und Walthers von der Vogelweide. Überlegungen zur Absage an das Tagelied der hochhöfischen Zeit. In: Krüger, Rüdiger - Kühnel, Jürgen - Kuolt, Joachim (hrsg.): Ist zwîvel herzen nâchgebûr. Günther Schweikle zum 60. Geburtstag. Stuttgart: Helfant-Ed., S. 63-82 (hier S. 66). 
Die Präteritumformen (troug, wânde, tagte) versetzen die Szene in die Vergangenheit, was die Intensität des Tagelied-Erlebnisses relativiert. Die Strophe wird mit der Tagesanbruchsformel („do tagt ez“12) abgeschlossen.

In der zweiten Strophe kommt die weibliche Stimme zu Wort, welche ebenso ein vergangenes Tageliederlebnis beschreibt („klage pflac“, „bî mir lag“). Das Schema der ersten Strophe wird nachgebildet, indem die Betrachtungsweise vom Subjekt ausgeht und auf das Objekt gerichtet wird. Während sich die männliche Stimme der ersten Strophe auf das Visuelle konzentriert, wird von der weiblichen Stimme das Handeln vor Augen geführt (klagen). Wie in der ersten Strophe kommt am Anfang die auf die Zukunft projizierte Wunschvorstellung vor (Refrain 1) („sol aber iemer mê // den morgen hie betagen“) und die Strophe wird mit Refrain 2 („Dô tagte ez“) abgeschlossen. Die gemeinsame Vergangenheit soll als Versprechen für die gemeinsame Zukunft dienen. Die repetitive Form erzeugt eine Scheinresponsivität, welche die mentale Nähe betont.

In der vorletzten Strophe wird das statische Bild der ersten Strophe durch aktives Handeln ersetzt: Der Sänger beschreibt die Folgen der drohenden Trennung (trehene - weibliche Domäne) und das (aktive) Trösten der Frau (männliche Domäne). Dabei lässt sich das Trösten der Dame als eine Reaktion auf ihre Klage in der vorangehenden Strophe lesen. ${ }^{13}$ Die letzte Strophe greift das Körper-Bild der Eingangsstrophe auf. Die weibliche Stimme bestätigt die Authentizität der männlichen Aussage, indem sie sich explizit mit dem leuchtenden Objekt (sunder wât) identifiziert.

Es ist vorausgeschickt worden, dass im diskutierten Lied Gattungen kombiniert werden, welche bezüglich der Raum-Zeit-Perspektive unterschiedlich zu verorten sind. Das Tagelied setzt das Miteinander, die physische Kopräsenz der Partner im Gespräch, voraus, die Zeitebene, welche am Bildhaftesten in Wolframs Tönen zum Vorschein kommt, ist nun die Gegenwart. Der Wechsel, hingegen, präsumiert eine räumliche Trennung (die Absenz) der Partner, was eine längere Zeitspanne in Anspruch nimmt (hier und jetzt plus die Vergangenheit). Daraus ergibt sich häufig eine scheinbare Inkohärenz ${ }^{14}$, die Inhalte sind nicht streng organisiert, die Aussagen schweben in Leerräumen. In den klassischen Repräsentanten der Gattung werden häufig Situationen wiedergegeben, deren Gültigkeit im Moment der Rede umstritten ist (Kommunikationsstörung ist ein konstitutives Element des Wechsels). Morungen schafft keinen reinen Wechsel. In dem betreffenden Ton handelt es sich nämlich um keine Selbstgespräche, welche die Liebe aus unterschiedlichen Perspektiven thematisieren, sondern um einen Diskurs, der „der gemeinsamen Erlebnissphäre"15 entspringt und dementsprechend inhaltlich eindeutig

12 Die Präsensform kommt nur in der ersten Strophe vor. In den Editionen wird eine Konjunktur vorgenommen, es ist aber durchaus plausibel, dass der Sänger der ersten Strophe das Bild im Hintergrund der Morgendämmerung aus der Erinnerung ruft.

13 Möckel, Sebastian (2011): „Der süeze wechsel under zwein.“ Intime Dialoge im mittelhochdeutschen Tagelied um 1200. In: Münkler, Martina (hrsg.): Aspekte einer Sprache der Liebe. Formen des Dialogischen im Minnesang. Frankfurt am Main: Peter Lang, S. 127-156 (hier S. 138).

14 „scheinbar“, weil die Definition von Kohärenz den Vorstellungen nicht entsprechen muss.

15 Tubach Frederic C. (1960): Wechselform und Tageliedsituation in dem Tageliedwechsel 143,22. In: Zeitschrift für deutsches Altertum und deutsche Literatur 79, S. 309-315 (hier S. 313). 
fixiert ist. Infolge des hohen Grades an Responsivität konkurriert das Lied mit Dialogliedern, deren Status eine Harmonie in der Beziehung ermöglicht: Analoge Strukturen, Wiederaufnahmen von Leitbegriffen, motivische Verknüpfung, zirkulärer Schluss funktionieren als Mittel dazu. ${ }^{16}$ Die Dringlichkeit der Morgendämmerung geht im Aneinandervorbeireden des Wechsels nicht völlig verloren: Das Momenthafte avanciert mittels der Form: Das Enjambement (I, 3) illustriert die ungünstigen Zeitverhältnisse.

Infolge der konsequenten Verlagerung des Tagelied-Erlebnisses in die Vergangenheit wird jedoch der Rahmen des Wechsels nicht völlig gesprengt. Die Raumverhältnisse werden komplexer als im Tagelied, indem der Raum der Erinnerung (das Tagelied-Erlebnis) mit dem Raum des Erinnerns (hier und jetzt) korreliert. Dies öffnet neue Deutungsmöglichkeiten: Das Tagelied wird in doppelte Räume verlegt und dementsprechend entzeitlicht, ${ }^{17}$ wodurch die Intensität des Erlebnisses und gleichzeitig dessen Extensität (Möglichkeit der Wiederholbarkeit) ausgespielt werden. Ein one-night-stand-Erlebnis, welches bei anderen Tageliedern im Spiel ist, kommt nicht in Frage, was die im Kontext des klassischen Minnesangs eher problematische Gattung dem Ethos der Hohen Minne annähert. Die gegenseitige Zuneigung, welche sowohl auf der formalen als auch auf der inhaltlichen Ebene erkennbar ist, hebt die, für beide Gattungen konstitutive, Rollenasymmetrie auf. ${ }^{18}$

Die Ich-Perspektiven, sowohl die männliche als auch die weibliche, sind hier gleichmäBig austariert und tragen zur Objektivierung der Schilderung bei. ${ }^{19}$

\section{Tagelied-Pastourelle: Šla dva tovařišě}

Im Folgenden wende ich mich dem Mischtyp Tagelied-Pastourelle zu, welches am Beispiel des alttschechischen Liedes Šla dva tovařišě besprochen wird. Die Montage enthält zwei Liedtypen, welche gattungskonstitutive Gemeinsamkeiten nachweisen: Beide stellen Repräsentanten des sog. genre objectif ${ }^{20}$ dar, die durch dialogische Form geprägt sind. Auch thematisch sind sie sich sehr nah, indem die Begegnung eines Ritters mit a) einer Dame und b) einer Hirtin beschrieben wird. Der unterschiedliche gesellschaftliche Status der Frau bedingt indes eine unterschiedliche Raumzuschreibung: Während sich das Tagelied-Erlebnis in einer Kemenate abspielt, bewegt man sich in der Pastourelle im Freien.

16 Möckel [Anm. 13], S. 138.

17 Möckel [Anm. 13], S. 139.

18 Hier polemisiere ich mit Möckel, der auf eine genderspezifische Asymmetrie hinweist. Im Vergleich mit dem für das Tagelied typischen Null-Engagement des männlichen Partners kommt es hier zu einer Emanzipation zugunsten der Aktivität. Das obligate „Schatz ich muss zum Bus“ kommt hier nicht vor (vgl. Kohlschmidt, Werner - Mohr, Wolfgang (2001): Reallexikon der deutschen Literaturgeschichte. Band 1, A-K. 2. Auflage. Berlin - New York: De Gruyter, S. 349.).

19 Mertens, Volker (1988): Erzählerische Kleinformen. Die genres objectifs im deutschen Minnesang: „Fragmente eines Diskurses über die Liebe“. In: Grubmüller, Klaus - Johnson, Peter - Steinhoff, Hans-Hugo (hrsg.): Kleine Erzählformen im Mittelalter. Padeborner Colloquium 1987. Paderborn - München - Wien - Zürich: Schöningh, S. 49-65 (hier S. 55).

20 Schweikle, Günther (1995): Minnesang. 2. Aufl. Stuttgart: Metzler, S. 142. 
Die Gattung Pastourelle, welche im romanischen Raum häufig vertreten ist, erscheint im deutschen Minnesang in reiner Form nur einmal. ${ }^{21}$ Es gibt jedoch Texte, die Anklänge an die Pastourelle enthalten. ${ }^{22}$ Ein Paradebeispiel liefert das bekannteste Lied Walthers: das Lindenlied.

Das anonyme alttschechische Lied kann formal in zwei Teile gegliedert werden: In den ersten 10 Versen wird die Einbettung im Freien von einem Erzähler-Ich beschrieben. Die für die Pastourelle typische Eröffnung mit einem locus amoenus signalisiert eingangs die Gattungszugehörigkeit. Jedoch fehlt ein relevantes gattungskonstitutives Signal, nämlich eine vordefinierte Standeszugehörigkeit. Die Liebhaber („tovařišě“, Gesellen; Z. 1) werden auch im späteren Verlauf des Liedes standesgemäß nicht eingeordnet. Eine Zäsur bildet Zeile 10 (etwa die Hälfte des Tons), welche eine konkrete Zeitangabe enthält (bis 3 Uhr habe man das Liebesspiel getrieben ${ }^{23}$ ). Die Morgendämmerung, welche das klassische Tagelied prägt, wird nun nicht durch akustische oder aber optische Signale eingeführt - was sich in der freien Natur angeboten hätte -, sondern mittels einer Zeitangabe. Im zweiten Teil sprechen männliche und weibliche Stimmen abwechselnd im Dialog. Auf die Aufforderung des Mannes in der dritten Strophe, sich nochmals auf das Liebesspiel einzulassen (hier stark euphemistisch ausgedrückt, III, 3), muss die Frau ablehnend reagieren. Der Grund des notwendigen Abschieds (III, 6), der Tagesanbruch, welcher dem aufmerksamen Hörer/ Leser bereits bekannt ist, wird in der vierten Strophe von der Mannesstimme (!) artikuliert (,již denička zchodí“; IV, 1). Die logische Konsequenz des Tageliedes wird hier nun umgekehrt: Zuerst wird die Notwendigkeit des Abschieds von der Frau erwähnt, erst dann wird diese durch das optische Signal (der Morgenstern) bestätigt. Durch die doppelte Bestätigung wird das obligate Instrumentarium des Tageliedes ins Spiel gebracht. Die durch den sich immer akuter nähernden Abschied bedingte Trauer lässt sich nur mittels einer auf die Zukunft projizierten Wunschvorstellung mildern: Ein nächstes Beisammensein, dessen Realisierung fast als Versprechen formuliert ist, erscheint nun als plausibel („skuoro-li sě vrátíš $\mathrm{V}, 3$; ,ját sě k tobě vrátím // na každú hodinu“ (für immer) VI, 3). Die Prägnanz des Versprechens relativiert wiederum die Einmaligkeit des Tagelied-Erlebnisses.

Die Raumzuschreibung, welche bezüglich der Bestimmung der Gattungszugehörigkeit ausschlaggebend ist, stellt für die Forschung ein Rätsel dar. Die diversen Signale führten zu bizarren Vorschlägen. Eine derartig obskure Interpretation bietet Lehár ${ }^{24}$ an, der das Geschehen in ein Freudenhaus platziert. Als Hauptargument dient ihm die Tatsache, dass die zwei Liebhaber von einer dritten Instanz, welche man in einer Naturszenerie ausschließen müsste, gebettet wurden (II,2). Die finale Hoffnung des Mannes, dass sich die beiden wieder auf grünem Gras treffen (VI,2) wird als (bloß auf die

21 Schweikle [Anm. 20], S. 142.

22 Zu Pastourelle-Motiven im Leich vgl. Hübner, Gert (2008): Minnesang im 13. Jh. Tübingen: Gunter Narr, S. 99-116.

23 Das alttschechische Verb hráti ist polysemisch, eine Assoziation mit höfischen Turnieren bietet sich an (wetteifern).

24 Lehár, Jan (1982): K problematice staročeské písně Šla dva tovařišě. In: Česká literatura, Bd. 30, Nr. 5, S. 407-410. 
Farbe reduzierte) erotische Metapher abgetan. Ich schließe mich jedoch Všetička ${ }^{25}$ an, der auf Diskrepanzen hingewiesen hat: Er stellt sich beispielsweise die Frage, wieso die Liebhaber in das Freudenhaus, in welchem nach der gemeinsam verbrachten Nacht nur die Frau bleibt, gemeinsam schreiten. ${ }^{26}$ Grünes Gras interpretiere ich dementsprechend als eine traditionelle, erotische Spannung erzeugende, Komponente der Naturdichtung, welche den besprochenen Ton formal einrahmt. Darüber hinaus kann die grüne Farbe den Anfang der Liebe markieren. Die enigmatische Einbettung muss nicht durch einen menschlichen Aktanten durchgeführt worden sein, sondern eher als Engagement der Natur angesehen werden. Die Intention, das Lied als derbe Poesie herabzusetzen, finde ich fehl am Platz. Die inhaltlichen Signale erweisen sich als unzulänglich, die formale Präzision des Liedes erinnert eher an die Virtuosität ${ }^{27}$ des Hohen Sanges.

\section{Fazit}

Es ist einer der Verdienste der mediävistischen Literaturwissenschaft, die heuristischen Theoriemodelle anhand des mittelalterlichen Textkorpuses zu hinterfragen. Die bislang reichlich rezipierte Bachtinsche Theorie, insbesondere das Phänomen der Dialogizität im Roman, stellten ein Beispiel dar. Ihre Postulate wurden in erster Linie im Bereich der Epik angewandt ${ }^{28}$, jedoch blieben diese in der Lyrik nicht ohne Anwendung. ${ }^{29}$

Die analysierten Texte repräsentieren Beispiele von Montagen ${ }^{30}$, welche durch eine Redevielfalt geprägt sind. Die Stimmen sind der auktorialen Autorität nicht untergeordnet, die Redeweisen und Topoi verweisen auf jeweils unterschiedliche Gattungen. Dadurch entsteht nicht nur auf der intratextuellen Ebene eine dialognahe Situation im Bachtinschen Sinne, sondern es werden, auf der extratextuellen Ebene, zwei Systeme in einem Dialog miteinander konfrontiert.

25 Všetička, František (1989): K staročeské písni Šla dva tovařišě. In: Listy filologické 112, 3, S. 168-171.

26 Lehár reagiert auf die Vorwürfe Všetičkas, seine ergänzende Erklärung, dass sich die zwei Liebenden vor dem Freudenhaus getroffen hätten und dann gemeinsam reingeschritten seien, erinnert mich eher an das heutige Amsterdam als an die Paradigmen der mittelalterlichen Lyrik. Dazu Lehár, Jan (1989): Odpověd' Františku Všetičkovi. In: Listy filologické 112, S. 172-173, hier 173.

$27 \mathrm{Zu}$ formalen Leistungen s. Všetička [Anm. 25], S. 170.

28 Dazu z. B. Kasten, Ingrid (1995): Bachtin und der höfische Roman. In: Lindemann, Dorothee - Volkmann, Berndt - Wegera, Klaus-Peter (hrsg.): Bickelwort und wildiu mære. Festschrift für Eberhard Nellmann zum 65. Geburtstag (Göppinger Arbeiten zur Germanistik 618). Göppingen: Kümmerle, S. 51-70.

29 Dazu Lachmann Renate (1983): Dialogizität und poetische Sprache. In: Lachmann, Renate (hrsg.): Dialogizität. München: Fink, S. 51-62. Im Aufsatz kommt Lachmann zu dem Schluss, dass die von Bachtin ausschließlich dem Roman zugeschriebene Dialogizität für den Bereich der Lyrik von Interesse sein kann. M. Kern hat diese Annahme plausibilisiert, indem er Dialogizität und Hybridität von lyrischen Texten auf den Bereich der späthöfischen Lyrik angewandt hat; vgl. Kern, Manfred (2005): Hybride Texte - wilde Theorie? Perspektiven und Grenzen einer Texttheorie zur spätmittelalterlichen Liebeslyrik. In: Hübner, Gert (hrsg.): Deutsche Liebeslyrik im 15. und 16. Jahrhundert (Chloe, Beihefte zum Daphnis 37), S. 11-45.

30 Zum Begriff Montage vgl. Kern [Anm. 29], S. 25. 
Es sei hier eine These aufgestellt, dass diese durch die zweifache Kodierung entstandene Doppelsinnstruktur, eine textkonstitutive, textinterne Instanz ${ }^{31}$ darstellt, welche nicht nur den späten Minnesang prägt, sondern bereits in der Phase vorkommt, in welcher das System allmählich konsolidiert worden ist. Diese doppelte Kodierung ermöglicht, dass in den Texten gleichzeitig zentrifugale und zentripetale Kräfte wirken, welche das Lied hinsichtlich des konventionellen Sanges (de)zentralisieren.

\section{Literatur}

Bachtin Michail (1979): Die Ästhetik des Wortes (hrsg. von Rainer Grübel). Frankfurt am Main: Suhrkamp.

Backes, Martina (hrsg.) (1992): Tagelieder des deutschen Mittelalters. Stuttgart: Reclam.

Bennewitz Ingrid (1993): Neidhart. Wie sol ich die bluomen überwinden. In: Tervooren, Helmut (hrsg.): Gedichte und Interpretationen. Stuttgart: Reclam, S. 321-338.

Eco, Umberto (1979): The Role of the Reader. Explorations in the Semiotics of Texts. Bloomington: Indiana University Press.

Eikelmann, Manfred (1999): Dialogische Poetik. Zur Kontinuität älterer poetologischer Traditionen des Minnesangs am Beispiel des Wechsels. In: Cramer, Thomas - Kasten, Ingrid (hrsg.): Mittelalterliche Lyrik. Probleme der Poetik, S. 85-106.

Gottzmann, Carola L. (1989): Das Tagelied Heinrichs von Morungen und Walthers von der Vogelweise. Überlegungen zur Absage an das Tagelied der hochhöfischen Zeit. In: Krüger, Rüdiger - Kühnel, Jürgen - Kuolt, Joachim (hrsg.): Ist zwîvel herzen nâchgebûr. Günther Schweikle zum 60. Geburtstag. Stuttgart: Helfant-Ed., S. 63-82.

Hübner, Gert (2008): Minnesang im 13. Jh. Tübingen. Gunter Narr.

Jauß, Hans Robert (1972): Theorie der Gattungen und Literatur des Mittelalters, In: Jauß, Hans Robert - Köhler Erich (hrsg.): Grundriss der romanischen Literaturen des Mittelalters, Bd. 1. Heidelberg, S. 107-138.

Kasten, Ingrid (1995): Bachtin und der höfische Roman. In: Lindemann, Dorothee - Volkmann, Berndt - Wegera, Klaus-Peter (hrsg.): Bickelwort und wildiu mære. Festschrift für Eberhard Nellmann zum 65. Geburtstag (Göppinger Arbeiten zur Germanistik 618). Göppingen: Kümmerle, S. 51-70.

Kasten, Ingrid (1986): Frauendienst bei Trobadours und Minnesängern im 12. Jahrhundert. Heidelberg: Carl Winter Universitätsverlag.

Kern, Manfred (2005): Hybride Texte -wilde Theorie? Perspektiven und Grenzen einer Texttheorie zur spätmittelalterlichen Liebeslyrik. In: Hübner, Gert (hrsg.): Deutsche Liebeslyrik im 15. und 16. Jahrhundert (Chloe, Beihefte zum Daphnis 37), S. 11-45.

Kerth, Sonja (2007): „Jô enwas ich niht ein eber wilde“. Geschlechterkonzeptionen im „Wechsel“. In: Zeitschrift für deutsches Altertum und deutsche Literatur 123, S. 143-161.

Kiening, Christian (1998): Schwierige Modernität. Der „Ackermann“ des Johannes von Tepl und die Ambiguität historischen Wandels. Tübingen: Niemeyer.

Kohlschmidt, Werner - Mohr, Wolfgang (2001): Reallexikon der deutschen Literaturgeschichte. Band 1, A-K. 2. Auflage. Berlin - New York: De Gruyter.

31 Den impliziten Hörer/ Autor verstehe ich als textinterne Instanzen, vgl. Lachmann [Anm. 29], S. 53. 
Lachmann Renate (1983): Dialogizität und poetische Sprache. In: Lachmann, Renate (hrsg.): Dialogizität. München: Fink, S. 51-62.

Lehár, Jan (1982): K problematice staročeské písně Šla dva tovařǐ̌ě. In: Česká literatura, Bd. 30, Nr. 5, S. 407-410.

Lehár, Jan (1989): Odpověd’ Františku Všetičkovi. In: Listy filologické 112, S. 172-173.

Mertens, Volker (1988): Erzählerische Kleinformen. Die genres objectifs im deutschen Minnesang: „Fragmente eines Diskurses über die Liebe“. In: Grubmüller, Klaus - Johnson, Peter - Steinhoff, Hans-Hugo (hrsg.): Kleine Erzählformen im Mittelalter. Padeborner Colloquium 1987. Paderborn - München - Wien - Zürich: Schöningh, S. 49-65.

Möckel, Sebastian (2011): „Der süeze wechsel under zwein.“ Intime Dialoge im mittelhochdeutschen Tagelied um 1200. In: Münkler, Martina (hrsg.): Aspekte einer Sprache der Liebe. Formen des Dialogischen im Minnesang. Frankfurt am Main: Peter Lang, S. 127-156.

Münkler, Martina (hrsg.) (2011): Aspekte einer Sprache der Liebe. Formen des Dialogischen im Minnesang. Frankfurt am Main: Peter Lang.

Schnell, Rüdiger (1999): Frauenlied, Manneslied und Wechsel im deutschen Minnesang. Überlegungen zu „gender“ und Gattung. In: Zeitschrift für deutsches Altertum und deutsche Literatur 128, S. 127-180.

Schweikle, Günther (1995): Minnesang. 2. Aufl. Stuttgart: Metzler.

Stanovská Sylvie (2015): Sagt mir jemand, was Liebe ist? Deutschsprachige und tschechische Liebeslyrik des Mittelalters. Eine Typologie. Brno: Muni Press.

Tervooren, Helmut (hrsg.) (1993): Gedichte und Interpretationen. Mittelalter. Stuttgart. Reclam.

Tubach Frederic C. (1960): Wechselform und Tageliedsituation in dem Tageliedwechsel 143,22. In: Zeitschrift für deutsches Altertum und deutsche Literatur 79, S. 309-315.

Všetička, František (1989): K staročeské písni Šla dva tovařišě. In: Listy filologické 112, 3, S. 168-171. 


\section{Anhang}

\section{Šla dva tovařišě 32}

I.

Šla dva tovařišě

$\mathrm{v}$ jedno miesto tíšè.

Es gingen zwei Liebende

Míle jě přivítali, dobrá slova dali.

II.

Dobrá slova davše

dobřě položili, jednoho na ruce, heimlich zu einem Ort.

Dort wurde Ihnen ein lieber Empfang bereitet mit guten Worten.

Nachdem gute Worte gewechselt waren, wurden sie gut gebettet, druhého na loži. sie auf den Händen, er auf dem Lager.

III.

Tut’ sta spolu hrála Dort betrieben sie das Liebesspiel do třetie hodiny: Bis zur dritten Stunde:

„Obrátiž sě ke mně svým líčkem červeným!“

„Wende zu mir „Tohot neučiním, musíš pryč ode mne.“ IV. „Již denička zchodí, musím pryč od tebe. deine roten Wangen!“ „Das tue ich nicht, du musst fort von mir,“

Jižt musím od tebe s velikú žalostí.“

$\mathrm{V}$.

„Rač tě Buoh žehnati muoj milý pane!

Skuoro-li sě vrátís muoj milý pane?“

VI.

„Ját sě k tobě vrátím na zelenú trávu, ját sě k tobě vrátím na každú hodinu.“

„Der Morgenstern geht schon auf, ich muss fort von dir. schon muss ich weg von dir in großem Leid.“
„Möge dich Gott segnen, mein lieber Herr!
Kommst du bald wieder, mein lieber Herr?"
Ich komme wieder zu dir auf das grüne Gras.
Ich komme wieder zu dir für immer.

\footnotetext{
32 Altschechischer Text sowie Übersetzung zitiert nach: Stanovská, Sylvie (2015): Sagt mir jemand, was Liebe ist? Deutschsprachige und tschechische Liebeslyrik des Mittelalters. Eine Typologie. Brno: Masarykova univerzita, Zitiert und übersetzt nach Stanovská, S. 278-279.
} 
Mgr. Kristýna Solomon, Ph.D. / kristyna.solomon@upol.cz

Univerzita Palackého, Filozofická fakulta, Katedra germanistiky

Křížkovského 10, 77180 Olomouc, CZ

This work can be used in accordance with the Creative Commons BY-SA 4.0 International license terms and conditions (https://creativecommons.org/licenses/by-sa/4.0/legalcode). This does not apply to works or elements (such as image or photographs) that are used in the work under a contractual license or exception or limitation to relevant rights 
